\title{
The Combined RKM and ADM for Solving Nonlinear Weakly Singular Volterra Integrodifferential Equations
}

\author{
Xueqin Lv and Sixing Shi \\ School of Mathematics and Sciences, Harbin Normal University, Heilongjiang, Harbin, 150025, China \\ Correspondence should be addressed to Xueqin Lv, hashidalvxueqin@126.com
}

Received 2 July 2012; Accepted 12 November 2012

Academic Editor: Natig M. Atakishiyev

Copyright (C) 2012 X. Lv and S. Shi. This is an open access article distributed under the Creative Commons Attribution License, which permits unrestricted use, distribution, and reproduction in any medium, provided the original work is properly cited.

The reproducing kernel method (RKM) and the Adomian decomposition method (ADM) are applied to solve $n$ th-order nonlinear weakly singular Volterra integrodifferential equations. The numerical solutions of this class of equations have been a difficult topic to analyze. The aim of this paper is to use Taylor's approximation and then transform the given $n$ th-order nonlinear Volterra integrodifferential equation into an ordinary nonlinear differential equation. Using the RKM and ADM to solve ordinary nonlinear differential equation is an accurate and efficient method. Some examples indicate that this method is an efficient method to solve $n$ th-order nonlinear Volterra integro-differential equations.

\section{Introduction}

In this paper, we consider the following $n$ th-order nonlinear weakly singular Volterra integrodifferential equation of the following form [1-4]:

$$
\sum_{i=0}^{n} a_{i}(x) u^{(i)}(x)=f(x)+\int_{a}^{x} K(t, x) u^{m}(t) d t, \quad a \leqslant x \leqslant b,
$$

where $m>1, f(x)$ is a given function, $u(x)$ is the unknown function, and $K(t, x)$ is the kernel of the integro equation. We usually assume that the function $u(x)$ and $f(x)$ are continuous or square integrable on $[a, b]$.

Some problems of mathematical physics are described in terms of (1.1) which has been studied by different methods including the spline collocation method [5], piecewise polynomials [6], Haar wavelets [7], the homotopy perturbation method (HPM) [8, 9], 
the wavelet-Galerkin method [10], Taylor polynomials [11], the Tau method [12], the sinc-collocation method [13], the combined Laplace transform-Adomian decomposition method [14], and the Adomian's asymptotic decomposition method [15] to determine exact and approximate solutions. But to our knowledge there is still no viable analytic approach for solving weakly singular Volterra integro-differential equations. The present work is motivated by the desire to obtain approximate solution to $n$ th-order nonlinear weakly singular Volterra integro-differential equation, where the integrand is weakly singular in the sense that its integral is continuous at the singular point, that is, its kernel $K(t, x)=1 /(x-t)^{\alpha}$ is singular as $t \rightarrow x$.

Reproducing kernel theory has important application in numerical analysis, differential equation, probability and statistics and so on $[16,17]$. And the RKM has been applied successfully to solving linear and nonlinear problems [18-20].

The rest of the paper is organized as follows. In the Section 2, transforming (1.1) into an differential equation by Taylor's approximation. In Section 3, the RKM is introduced. Applying RKM and ADM to solving (1.1) is discussed in Section 4. The numerical examples are presented in Section 5. Finally, a brief conclusion is stated in last section.

\section{Taylor's Approximation}

Consider the following $n$ th-order nonlinear weakly singular Volterra integro-different equation:

$$
\sum_{i=0}^{n} a_{i}(x) u^{(i)}(x)=f(x)+\int_{a}^{x} \frac{F(u(t))}{(x-t)^{\alpha}} d t, \quad a \leqslant x \leqslant b, 0<\alpha<1 .
$$

We have by setting

$$
\begin{gathered}
y(t)=F(u(t)), \\
\sum_{i=0}^{n} a_{i}(x) u^{(i)}(x)=f(x)+\int_{a}^{x} \frac{y(t)}{(x-t)^{\alpha}} d t, \quad a \leqslant x \leqslant b, 0<\alpha<1 .
\end{gathered}
$$

Rewriting (2.3) as

$$
\sum_{i=0}^{n} a_{i}(x) u^{(i)}(x)=f(x)+\int_{a}^{x} \frac{y(t)-y(x)+y(x)}{(x-t)^{\alpha}} d t
$$

where the solution under the integral has been replaced by $y(t)-y(x)+y(x)$. Thus,

$$
\sum_{i=0}^{n} a_{i}(x) u^{(i)}(x)=f(x)+y(x) \int_{a}^{x} \frac{1}{(x-t)^{\alpha}} d t+\int_{a}^{x} \frac{y(t)-y(x)}{(x-t)^{\alpha}} d t
$$


So that

$$
\sum_{i=0}^{n} a_{i}(x) u^{(i)}(x)=f(x)+y(x) \frac{(x-a)^{1-\alpha}}{1-\alpha}+\int_{a}^{x} \frac{y(t)-y(x)}{(x-t)^{\alpha}} d t
$$

or equivalently

$$
\sum_{i=0}^{n} a_{i}(x) u^{(i)}(x)=f(x)+y(x) \frac{(x-a)^{1-\alpha}}{1-\alpha}-\int_{a}^{x}(x-t)^{1-\alpha} \frac{y(t)-y(x)}{t-x} d t
$$

We use the following Taylor's approximation of degree $n$ of $y(t)$ about $t=x$ :

$$
y(t) \approx y(x)+(t-x) y^{\prime}(x)+\frac{(t-x)^{2}}{2 !} y^{\prime \prime}(x)+\cdots+\frac{(t-x)^{n}}{n !} y^{(n)}(x)
$$

Thus,

$$
y(t)-y(x) \approx(t-x) y^{\prime}(x)+\frac{(t-x)^{2}}{2 !} y^{\prime \prime}(x)+\cdots+\frac{(t-x)^{n}}{n !} y^{(n)}(x)
$$

Substituting the approximate relation (2.9) into the right hand side of (2.7) yields

$$
\sum_{i=0}^{n} a_{i}(x) u^{(i)}(x)=f(x)+\sum_{i=0}^{n}(-1)^{i} \frac{(x-a)^{i+1-\alpha}}{i !(i+1-\alpha)} y^{(i)}(x)
$$

Therefore, (2.1) can be approximated by the $n$ th-order nonlinear differential equation (2.10).

\section{Analysis of Reproducing Kernel Hilbert Space}

Definition 3.1 (reproducing kernel Space $W_{n+1}[a, b]$, see [17]).

$$
\begin{gathered}
W_{n+1}[a, b]=\left\{u(x) \mid u^{(n)}(x) \text { is an absolutely continuous real value function in }[a, b],\right. \\
\left.u^{(n+1)}(x) \in L^{2}[a, b], x \in[a, b]\right\}
\end{gathered}
$$

and endowed it with the inner product and norm, respectively,

$$
\langle u(x), v(x)\rangle_{W_{n+1}}=\sum_{i=0}^{n} u^{(i)}(a) v^{(i)}(a)+\int_{a}^{b} u^{(n+1)}(x) v^{(n+1)}(x) d x, \quad\|u\|_{W_{n+1}}=\langle u, u\rangle^{1 / 2}
$$


Theorem 3.2. The space $W_{n+1}[a, b]$ is a reproducing kernel space. That is, there exists a function $R_{x}(y)$, for each fixed $x \in[a, b], R_{x}(y) \in W_{n+1}[a, b]$, and for any $u(y) \in W_{n+1}[a, b]$, satisfying

$$
\left\langle u(y), R_{x}(y)\right\rangle_{W_{n+1}}=u(x),
$$

the reproducing kernel $R_{x}(y)$ can be denoted by

$$
R_{x}(y)= \begin{cases}\sum_{i=1}^{2 n+2} a_{i}(x) y^{i-1}, & y \leqslant x \\ \sum_{i=1}^{2 n+2} b_{i}(x) y^{i-1}, & y>x\end{cases}
$$

where $a_{i}(x), b_{i}(x)(i=1,2, \ldots, 2 n+2)$ are known coefficients.

Theorem 3.3. Let $W_{n+1}[a, b]$ be a reproducing kernel space and $u_{m}(x), u(x) \in W_{n+1}[a, b], m=1,2$, .... If $u_{m}(x)$ converges to $u(x)$ in the sense of $\|\cdot\|_{W_{n+1}}$, then $u_{m}^{(k)}(x)$ converges to $u^{(k)}(x)(k=0$, $1, \ldots, n)$ uniformly.

Property 1. If $W_{n+1}[a, b]$ is a reproducing kernel space, the reproducing kernel function $R_{x}(y)$ in $W_{n+1}[a, b]$ is unique.

Definition 3.4 (reproducing kernel Space $W_{1}[a, b]$, see [17]).

$$
\begin{gathered}
W_{1}[a, b]=\{u(x) \mid u(x) \text { is an absolutely continuous real value function in }[a, b] \\
\left.u^{\prime}(x) \in L^{2}[a, b], x \in[a, b]\right\}
\end{gathered}
$$

and endowed it with the inner product and norm, respectively,

$$
\langle u(x), v(x)\rangle_{W_{1}}=u(a) v(a)+\int_{a}^{b} u^{\prime}(x) v^{\prime}(x) d x, \quad\|u\|_{W_{1}}=\langle u, u\rangle^{1 / 2}
$$

There exists a unique reproducing kernel function $Q(x, y) \in W_{1}[a, b]$, and $Q(x, y)$ can be denoted by

$$
Q_{x}(y)= \begin{cases}1+y, & y \leqslant x \\ 1+x, & y>x\end{cases}
$$

The method of obtaining $Q_{x}(y)$, the coefficients of the reproducing kernel $R_{x}(y)$, and the proof of Theorems 3.1 and 3.2 are given in [17]. 


\section{Combined ADM and RKM}

\subsection{Representation of the Inverse Operator}

Here, we propose a new differential operator, as follows:

$$
\mathcal{L} u(x)=\sum_{i=0}^{n} a_{i}(x) u^{(i)}(x)
$$

letting

$$
N(x, u(x))=\sum_{i=0}^{n}(-1)^{i} \frac{(x-a)^{i+1-\alpha}}{i !(i+1-\alpha)} y^{(i)}(x),
$$

where $y(x)=F(u(x))$, then we convert (2.10) as follows:

$$
\mathcal{L} u(x)=f(x)+N(x, u(x)), \quad a \leqslant x \leqslant b .
$$

We now introduce how to determine the inverse operator $L^{-1}$ of $L$. Obviously, $\mathcal{L}$ : $W_{n+1}[a, b] \rightarrow W_{1}[a, b]$ is a bounded linear operator.

We choose $\left\{x_{i}\right\}_{i=1}^{\infty}$ as any dense set in $[a, b]$, and let $\psi_{x}(y)=\mathcal{L}^{*} Q_{x}(y)$, where $\mathcal{L}^{*}$ is the conjugate operator of $\mathcal{L}$ and $Q_{x}(y)$ is given by (3.7). Furthermore, for simplicity, let $\psi_{i}(x)$ denote $\psi_{x_{i}}(x)$, namely,

$$
\psi_{i}(x) \stackrel{\text { def }}{=} \psi_{x_{i}}(x)=\mathcal{L}^{*} Q_{x_{i}}(x)
$$

Now, several Lemmas are given.

Lemma 4.1. $\left\{\psi_{i}(x)\right\}_{i=1}^{\infty}$ is the complete system of $W_{n+1}[a, b]$.

Proof. For $u(x) \in W_{n+1}[a, b]$, let $\left\langle u(x), \psi_{i}(x)\right\rangle=0(i=1,2, \ldots)$, that is,

$$
\left\langle u(x), \mathcal{L}^{*} Q_{x_{i}}(x)\right\rangle=(\mathcal{L} u)\left(x_{i}\right)=0 .
$$

Note that $\left\{x_{i}\right\}_{i=1}^{\infty}$ is the dense set in $[a, b]$, therefore $(\mathcal{L} u)(x)=0$. It follows that $u(x)=0$ from the existence of $\mathcal{L}^{-1}$.

Lemma 4.2. The following formula holds

$$
\psi_{i}(x)=\left(\mathcal{L}_{\eta} R_{x}(\eta)\right)\left(x_{i}\right)
$$

where the subscript $\eta$ of operator $\mathcal{\perp}_{\eta}$ indicates that the operator $\perp$ applies to functions of $\eta$. 
Proof. Consider

$$
\begin{aligned}
\psi_{i}(x) & =\left\langle\psi_{i}(\xi), R_{x}(\xi)\right\rangle_{W_{n+1}[a, b]} \\
& =\left\langle\left(\mathcal{L}^{*} Q_{x_{i}}\right)(\xi), R_{x}(\xi)\right\rangle_{W_{n+1}[a, b]} \\
& =\left\langle Q_{x_{i}}(\xi),\left(\mathcal{L}_{\eta} R_{x}(\eta)\right)(\xi)\right\rangle_{W_{1}[a, b]} \\
& =\left(\mathcal{L}_{\eta} R_{x}(\eta)\right)\left(x_{i}\right) .
\end{aligned}
$$

This completes the proof.

The orthonormal system $\left\{\bar{\psi}_{i}(x)\right\}_{i=1}^{\infty}$ of $W_{n+1}[a, b]$ can be derived from Gram-Schmidt orthogonalization process of $\left\{\psi_{i}(x)\right\}_{i=1}^{\infty}$,

$$
\bar{\psi}_{i}(x)=\sum_{k=1}^{i} \beta_{i k} \psi_{k}(x), \quad\left(\beta_{i i}>0, i=1,2, \ldots\right),
$$

where $\beta_{i k}$ are orthogonal coefficients.

Theorem 4.3. If the inverse operator $L^{-1}$, exists and $\left\{x_{i}\right\}_{i=1}^{\infty}$ is dense on $[0,1]$, then the inverse operator $L^{-1}$ can be determined as

$$
u(x)=L^{-1}(f(x)+N(x, u(x)))=\sum_{i=1}^{\infty} B_{i} \bar{\psi}_{i}(x)
$$

where $B_{i}=\sum_{k=1}^{i} \beta_{i k}\left(f\left(x_{k}\right)+N\left(x_{k}, u\left(x_{k}\right)\right)\right)$.

Proof. From (4.9), it holds that

$$
\begin{aligned}
u(x) & =\sum_{i=1}^{\infty}\left\langle u(x), \bar{\psi}_{i}(x)\right\rangle_{W_{n+1}} \bar{\psi}_{i}(x) \\
& =\sum_{i=1}^{\infty} \sum_{k=1}^{i} \beta_{i k}\left\langle u(x), \mathcal{L}^{*} Q_{x_{k}}(x)\right\rangle_{W_{n+1}} \bar{\psi}_{i}(x) \\
& =\sum_{i=1}^{\infty} \sum_{k=1}^{i} \beta_{i k}\left\langle\mathcal{\perp} u(x), Q_{x_{k}}(x)\right\rangle_{W_{1}} \bar{\Psi}_{i}(x) \\
& =\sum_{i=1}^{\infty} \sum_{k=1}^{i} \beta_{i k}\left\langle f(x)+N(x, u(x)), Q_{x_{k}}(x)\right\rangle_{W_{1}} \bar{\psi}_{i}(x) \\
& =\sum_{i=1}^{\infty} \sum_{k=1}^{i} \beta_{i k}\left(f\left(x_{k}\right)+N\left(x_{k}, u\left(x_{k}\right)\right)\right) \bar{\psi}_{i}(x)
\end{aligned}
$$

The proof of the theorem is complete.

From Theorem 4.3, obviously, $L^{-1}$ is determined. 


\subsection{Decomposition Method}

By applying $L^{-1}$ to both sides of (4.3), we have

$$
u(x)=L^{-1} f(x)+L^{-1} N(x, u(x)) .
$$

The ADM introduces the solution $u(x)$ and the nonlinear function $N(x, u)$ by infinite series

$$
\begin{gathered}
u(x)=\sum_{i=0}^{\infty} u_{i}(x), \\
N(x, u(x))=\sum_{i=0}^{\infty} A_{i}(x),
\end{gathered}
$$

where $A_{n}$ are Adomian polynomials for the nonlinear term $N(x, u(x))$ and can be found from the following formula:

$$
A_{n}=\frac{1}{n !}\left[\frac{d^{n}}{d \lambda^{n}} f\left(\sum_{n=0}^{\infty} \lambda^{i} u_{i}\right)\right]_{\lambda_{0}}, \quad n \geqslant 0 .
$$

Substituting (4.12) and (4.13) into (4.11) yields

$$
\sum_{i=0}^{\infty} u_{i}(x)=L^{-1} f(x)+L^{-1} \sum_{i=0}^{\infty} A_{i}(x)
$$

According to the ADM, the components $u_{i}(x)$ can be determined as

$$
\begin{gathered}
u_{0}(x)=L^{-1} f(x), \\
u_{i+1}(x)=L^{-1} A_{i}(x), \quad i \geqslant 0,
\end{gathered}
$$

which gives

$$
\begin{gathered}
u_{0}(x)=\sum_{j=0}^{\infty} B_{0 j} \bar{\psi}_{j}(x), \\
u_{i+1}(x)=\sum_{j=0}^{\infty} B_{(i+1) j} \bar{\psi}_{j}(x), \quad i \geqslant 0,
\end{gathered}
$$

where $B_{0 j}=\sum_{k=1}^{j} \beta_{j k} f\left(x_{k}\right), B_{i j}=\sum_{k=1}^{j} \beta_{j k} A_{i-1}\left(x_{k}\right), i \geqslant 1$.

From (4.17), we can determine the components $u_{i}(x)$, and hence the series solution of $u(x)$ in (4.12) can be immediately obtained.

We obtain approximate solution of the following equation:

$$
U_{n}(x)=\sum_{i=0}^{n} u_{i}(x)
$$


The following use some examples to demonstrate the effectiveness of the algorithm.

\section{Numerical Examples}

To illustrate the applicability and effectiveness of our method, we consider the following examples. Symbolic and numerical computations performed by using Mathematica 5.0.

Example 5.1. Consider the following first-order nonlinear weakly singular Volterra integrodifferential equation:

$$
u^{\prime}(x)+p(x) u(x)=f(x)+\int_{a}^{x} \frac{F(u(t))}{(x-t)^{\alpha}} d t, \quad a \leqslant x \leqslant b, 0<\alpha<1
$$

with $\alpha=1 / 2, a=0, u(0)=0$ and $u(x)=x(x-1)$.

Let $F(u(x))=u^{2}, p(x)=(16 / 315) x^{5 / 2}(21+4 x(4 x-9))+1, f(x)=x^{2}+x-1$.

On $[0,1]$ select 100 points and get the approximate solution $U_{2}(x)=\sum_{n=0}^{2} u_{n}(x)$, the results are shown in Figure 1.

Example 5.2. Consider the following second-order nonlinear weakly singular Volterra integrodifferential equation:

$$
u^{\prime \prime}(x)+p(x) u^{\prime}(x)+q(x) u(x)=f(x)+\int_{a}^{x} \frac{F(u(t))}{(x-t)^{\alpha}} d t, \quad a \leqslant x \leqslant b, 0<\alpha<1
$$

with $\alpha=1 / 3, a=0, u(0)=u(1)=0$ and $u(x)=x(x-1)$.

$$
\text { Let } F(u(x))=u^{3}, p(x)=x^{2}+1, q(x)=(243 / 52360) x^{11 / 3}\left(-119+9 x\left(9 x^{2}-30 x+34\right)\right) \text {, }
$$
$f(x)=2 x^{3}+x^{2}+2 x+3$.

On $[0,1]$ select 100 points and get the approximate solution $U_{1}(x)=\sum_{n=0}^{1} u_{n}(x)$, the results are shown in Table 1.

Example 5.3. Consider the following third-order nonlinear weakly singular Volterra integrodifferential equation:

$$
u^{\prime \prime \prime}(x)+p(x) u^{\prime \prime}(x)+q(x) u^{\prime}(x)+r(x) u(x)=f(x)+\int_{a}^{x} \frac{F(u(t))}{(x-t)^{\alpha}} d t, \quad a \leqslant x \leqslant b, 0<\alpha<1
$$

with $\alpha=1 / 2, a=0, u(0)=u(1)=0, u^{\prime}(0)=0$ and $u(x)=x^{2}(x-1)$.

Let $F(u(x))=u^{2}, p(x)=x, q(x)=x-1, r(x)=(256 / 45045) x^{9 / 2}(143+20 x(6 x-13))$, $f(x)=3 x^{3}+x^{2}+6$.

On $[0,1]$ select 100 points and get the approximate solution $U_{2}(x)=\sum_{n=0}^{2} u_{n}(x)$, the results are shown in Figure 2.

\section{Conclusion}

In this paper, we have reduced the solution of nonlinear weakly singular Volterra integrodifferential equations to the solution of ordinary nonlinear differential equations by removing 


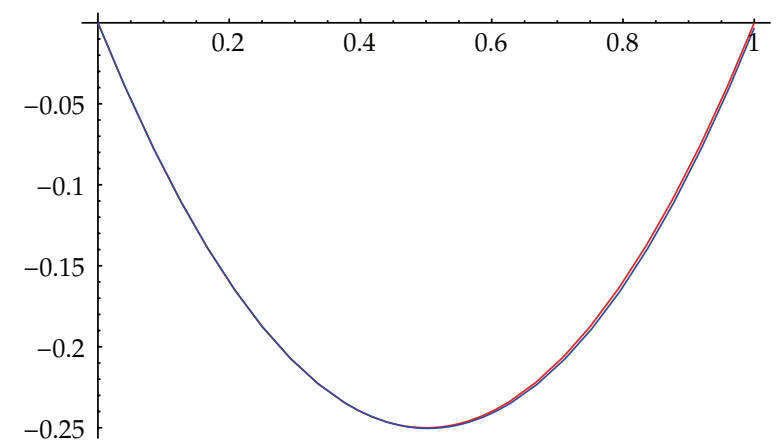

Figure 1: The approximate values $U_{2}(x)$ in comparison with its exact values $x(x-1)$.

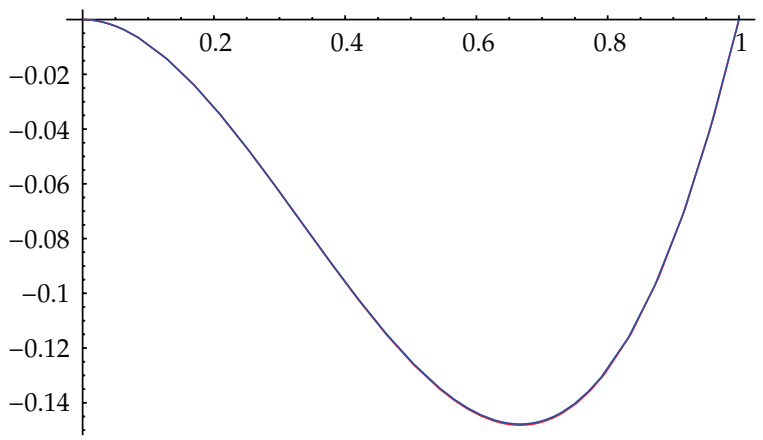

Figure 2: The approximate values $U_{2}(x)$ in comparison with its exact values $x^{2}(x-1)$.

Table 1: Comparison of the errors of $u(x)$ and $U_{1}(x)$.

\begin{tabular}{lccc}
\hline Node & Exact solution & Approximate solution & Relative error \\
\hline $1 / 100$ & -0.00980296 & -0.00980291 & $-5.55234 E-06$ \\
$11 / 100$ & -0.0970493 & -0.0970705 & $-2.18212 E-04$ \\
$21 / 100$ & -0.16469 & -0.164731 & $-2.50108 E-04$ \\
$31 / 100$ & -0.212724 & -0.212782 & $-2.70663 E-04$ \\
$41 / 100$ & -0.241153 & -0.241219 & $-2.72492 E-04$ \\
$51 / 100$ & -0.249975 & -0.250037 & $-2.44481 E-04$ \\
$61 / 100$ & -0.239192 & -0.239236 & $-1.84461 E-04$ \\
$71 / 100$ & -0.208803 & -0.208825 & $-1.04903 E-04$ \\
$81 / 100$ & -0.158808 & -0.158813 & $-2.96234 E-05$ \\
$91 / 100$ & -0.0892069 & -0.0892053 & $-1.80668 E-05$ \\
\hline
\end{tabular}

the singularity using an appropriate Taylor's approximation. Then we have demonstrated the solution of these ordinary nonlinear differential equations by RKM and ADM. The ADM is an accurate and efficient method to solve nonlinear weakly singular Volterra integro-differential equations. 


\section{Acknowledgments}

Research supported by the National Natural Science Foundation of China (61071181), the Educational Department Scientific Technology Program of Heilongjiang Province (12512133 and 12521148) and the Scientific Innovation Project for Graduate of Heilongiiang Province (YJSCX2012-184HLJ).

\section{References}

[1] J. Morchało, "On two-point boundary value problem for an integro-differential equation of second order," Polytechnica Posnaniensis, no. 9, pp. 51-66, 1975.

[2] R. P. Agarwal, "Boundary value problems for higher order integro-differential equations," Nonlinear Analysis, vol. 7, no. 3, pp. 259-270, 1983.

[3] A.-M. Wazwaz, "A reliable algorithm for solving boundary value problems for higher-order integrodifferentiable equations," Applied Mathematics and Computation, vol. 118, no. 2-3, pp. 327-342, 2001.

[4] E. Babolian and A. S. Shamloo, "Numerical solution of Volterra integral and integro-differential equations of convolution type by using operational matrices of piecewise constant orthogonal functions," Journal of Computational and Applied Mathematics, vol. 214, no. 2, pp. 495-508, 2008.

[5] H. Brunner, "On the numerical solution of nonlinear Volterra-Fredholm integral equations by collocation methods," SIAM Journal on Numerical Analysis, vol. 27, no. 4, pp. 987-1000, 1990.

[6] D. Conte and I. D. Prete, "Fast collocation methods for Volterra integral equations of convolution type," Journal of Computational and Applied Mathematics, vol. 196, no. 2, pp. 652-663, 2006.

[7] O. Lepik, "Haar wavelet method for nonlinear integro-differential equations," Applied Mathematics and Computation, vol. 176, no. 1, pp. 324-333, 2006.

[8] M. Ghasemi, M. Tavassoli Kajani, and E. Babolian, "Application of He's homotopy perturbation method to nonlinear integro-differential equations," Applied Mathematics and Computation, vol. 188, no. 1, pp. 538-548, 2007.

[9] J. Saberi-Nadjafi and A. Ghorbani, "He's homotopy perturbation method: an effective tool for solving nonlinear integral and integro-differential equations," Computers \& Mathematics with Applications, vol. 58, no. 11-12, pp. 2379-2390, 2009.

[10] Y. Mahmoudi, "Wavelet Galerkin method for numerical solution of nonlinear integral equation," Applied Mathematics and Computation, vol. 167, no. 2, pp. 1119-1129, 2005.

[11] K. Maleknejad and Y. Mahmoudi, "Taylor polynomial solution of high-order nonlinear VolterraFredholm integro-differential equations," Applied Mathematics and Computation, vol. 145, no. 2-3, pp. 641-653, 2003.

[12] G. Ebadi, M. Y. Rahimi-Ardabili, and S. Shahmorad, "Numerical solution of the nonlinear Volterra integro-differential equations by the tau method," Applied Mathematics and Computation, vol. 188, no. 2, pp. 1580-1586, 2007.

[13] M. Zarebnia and Z. Nikpour, "Solution of linear Volterra integro-differential equations via Sinc functions," International Journal of Applied Mathematics and Computer, vol. 2, pp. 1-10, 2010.

[14] A.-M. Wazwaz, "The combined Laplace transform-Adomian decomposition method for handling nonlinear Volterra integro-differential equations," Applied Mathematics and Computation, vol. 216, no. 4, pp. 1304-1309, 2010.

[15] L. Bougoffa, R. C. Rach, and A. Mennouni, “An approximate method for solving a class of weakly-singular Volterra integro-differential equations," Applied Mathematics and Computation, vol. 217, no. 22, pp. 8907-8913, 2011.

[16] H. Yao, The reserch of algorithms for some singular differential equations of higher even-order [Ph.D. thesis], Department of Mathematics, Harbin Institute of Technology, 2008.

[17] M. Gui and Y. Lin, Nonlinear Numercial Analysis in the Reproducing Kernel Space, Nova Science Publisher, New York, NY, USA, 2008.

[18] X. Lü and M. Cui, "Analytic solutions to a class of nonlinear infinite-delay-differential equations," Journal of Mathematical Analysis and Applications, vol. 343, no. 2, pp. 724-732, 2008.

[19] F. Geng and M. Cui, "New method based on the HPM and RKHSM for solving forced Duffing equations with integral boundary conditions," Journal of Computational and Applied Mathematics, vol. 233, no. 2, pp. 165-172, 2009.

[20] Z. Chen and Y. Lin, "The exact solution of a linear integral equation with weakly singular kernel," Journal of Mathematical Analysis and Applications, vol. 344, no. 2, pp. 726-734, 2008. 


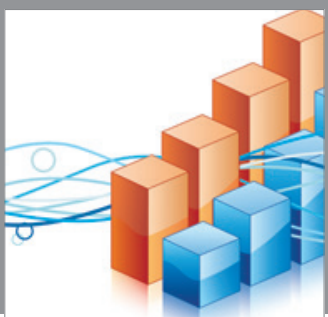

Advances in

Operations Research

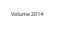

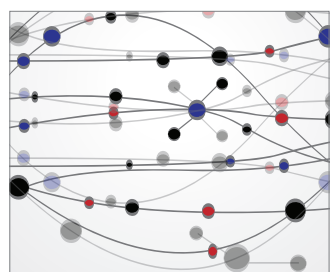

\section{The Scientific} World Journal
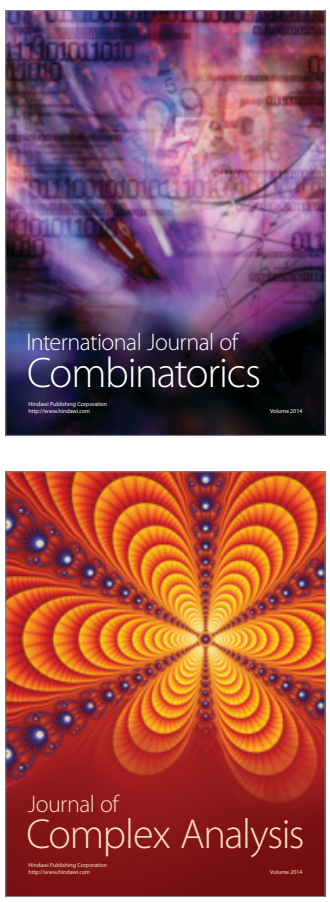

International Journal of

Mathematics and

Mathematical

Sciences
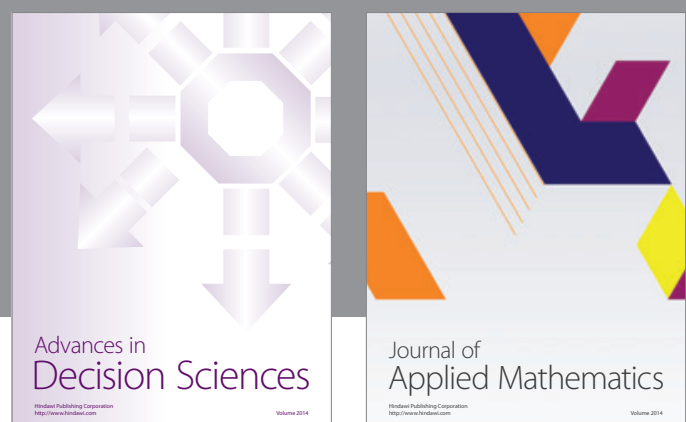

Journal of

Applied Mathematics
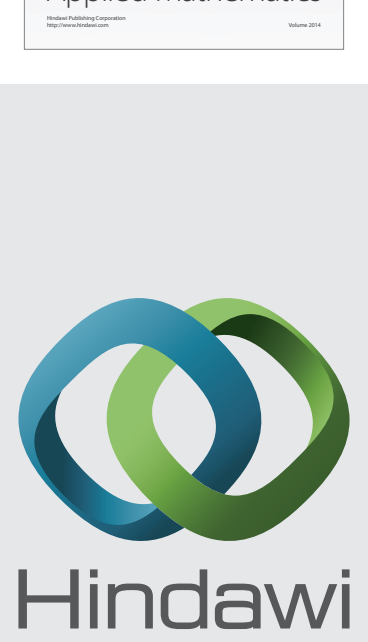

Submit your manuscripts at http://www.hindawi.com
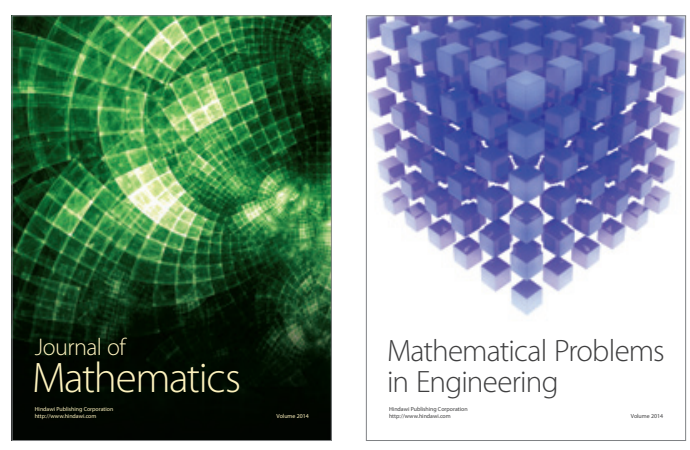

Mathematical Problems in Engineering
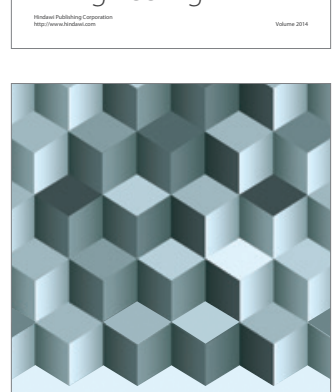

Journal of

Function Spaces
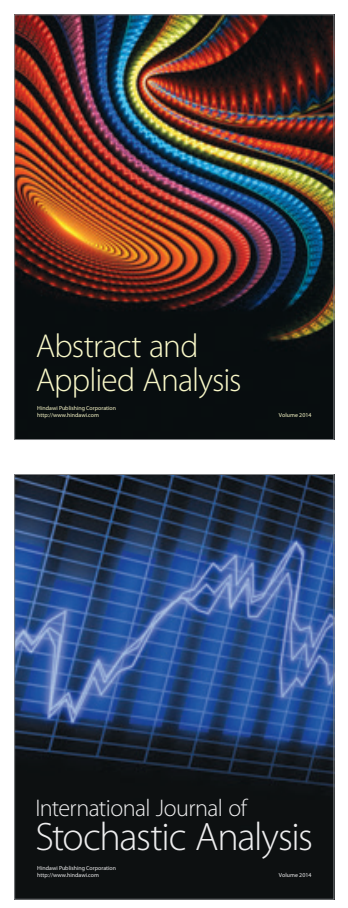

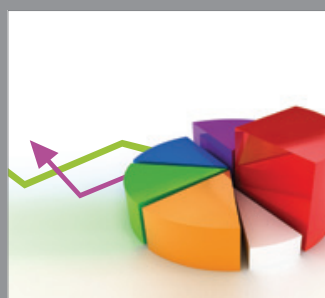

ournal of

Probability and Statistics

Promensencen
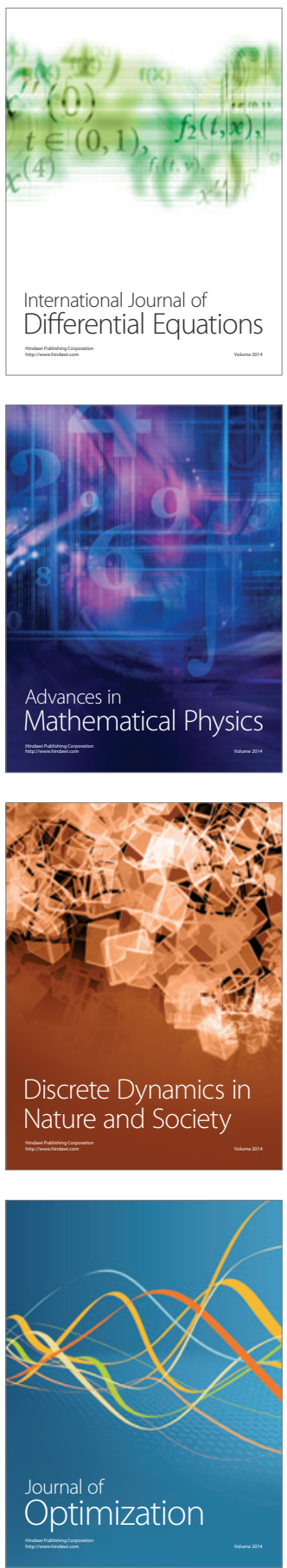